\title{
Modelling of Water-Saturated Grounds under a Curved Section of an Oil and Gas Pipeline
}

\author{
Tatyana Maltseva ${ }^{1, *}$ and Tatyana Saltanova ${ }^{2}$ \\ ${ }^{1}$ Industrial University of Tyumen, 625001 Volodarskogo str. 38, Tyumen, Russia \\ ${ }^{2}$ Tyumen State University, 625000 Semakova str. 10, Tyumen, Russia
}

\begin{abstract}
The paper studies the influence of the oil and gas pipeline on the base of water-saturated soil in terms of external forces effect (from the pipeline). The developed mathematical model of stress-strain state of "the oil and gas pipeline and the grounds" system allows us to identify the maximum load resulting from the impact of a curved section of the pipeline on the base as well as the deformations occurring in the grounds.
\end{abstract}

\section{Introduction}

Currently, transportation of oil is carried out by one of the most profitable modes of transport - by pipeline.

Its main advantage is a low cost of the transfer. But it has its drawbacks, the main of which is a one-time large capital investment in the construction, as before starting to use the oil pipeline you need to build it from the starting point to its destination.

During the operation, a number of factors influences the pipeline: the pressure caused by curvilinear sections of the pipeline, internal pressure, temperature fields of soil and the pumped medium, the pressure of the overlying soil layer, the deformation of the base associated with the differential settlement of soil under its freezing and thawing. The paper considers a factor of pressure of the pipeline section and the deformation of the base. As shown by calculations, the stresses associated with a curved pipeline axis may exceed the stresses created by the weight of the tube and the pumped product [1].

The biggest part of the territory of oil and gas transportation is made up of weak clay soils. A big number of ways to increase the bearing capacity of foundation soil have been developed and implemented [2-6].

The calculation of water-saturated base is carried out in the framework of the mathematical theory of water-saturated soil, with an account of the effect of residual excess pore pressure in a stable condition when the time factor is absent [7].

The three-dimensional problem of the "oil and gas pipeline + base" system is divided into two sub-problems in the two-dimensional formulations: one problem is related to the horizontal plane and is called the auxiliary one, the other problem deals with the vertical plane $x_{1} O x_{2}$, is called the principal one.

When addressing the auxiliary two-dimensional problem in the horizontal plane parallel to the ground surface one should define the longitudinal force q3 (with respect to the pipe

* Corresponding author: tgasu.maltseva.tatyana@,mail.ru 
axis) and transverse force $\mathrm{q} 2$. Then one solves the principal two-dimensional problem in the vertical plane, in which the horizontal force q2 is known as a result of the solution of the auxiliary problem. It is found with an account of the action of a longitudinal force q3. Thus, the solution of the three-dimensional problem (task) is replaced with the solution of two two-dimensional problems. Approximate taking account of the action of longitudinal force q3 is carried out through force q2.

\section{Problem formulation}

A mathematical model of vertical ultimate height (h) of a layer of water-saturated soil with an account of excessive residual pore pressure in a stable condition presents a system of differential equations with constant coefficients $G, \lambda, b$, c with respect to the displacement vector $\mathrm{u}=(\mathrm{u} 1, \mathrm{u} 2)$ of mineral soil particles [8]:

$$
\begin{gathered}
-\left(\left((G+\lambda) \frac{\partial \theta}{\partial x_{i}}+G \Delta u_{i}+b \frac{\partial^{2} u_{i}}{\partial x_{i}^{2}}+c \frac{\partial u_{i}}{\partial x_{i}}\right)\right)=F_{i}, i=1,2, \\
G=\frac{E_{S}}{2(1+v)}, \quad \lambda=\frac{E_{S} v}{(1+v)(1-2 v)}, \quad b=\frac{E_{l}}{\aleph^{2}}, \quad c=\frac{E_{l}}{\aleph h}, \quad \theta=\operatorname{div} \boldsymbol{u} .
\end{gathered}
$$

$E_{S}$ - modulus of deformation and $v$ - Poisson's ratio of mineral soil particles, $E_{l}$ mechanical constant of pore water, $\boldsymbol{N}$ - dimensionless quantity $(0<\aleph<1)$ determined from an experiment $[9 ; 10], h$ - the height of the compressible strata.

The boundary conditions for part of the boundary $S_{1}$ - kinematic, for part $S_{2}$ - static:

$$
\left.\boldsymbol{u}\right|_{S_{1}}=0,\left.\quad \boldsymbol{\sigma}\right|_{S_{2}}=\boldsymbol{q}\left(x_{1}, x_{2}\right),
$$

$\boldsymbol{\sigma}$ - stress tensor in the mineral particle of the soil with the effect of pore water being taken account of only in diagonal elements.

Excessive residual pore pressures vanish on the day $\left(x_{1}=0\right)$ surface of the body $S_{2}$ due to the draining coating and only the pressures in the mineral soil particles are left. Load $\boldsymbol{q}\left(\boldsymbol{x}_{1}, \boldsymbol{x}_{2}\right)$ - resultant of forces: $q_{1}$ - the weight per unit length of the pipeline with the product; horizontal force $q_{2}$ - the pressure of the curved section of the pipe on the ground. As already noted, it is defined with respect to the action of longitudinal force $q_{3}$.

Thus, the solutions of the two two-dimensional problems (the calculation of the curved section of the pipe and the calculation of the water-saturated soil base) have a common link, which is a transverse horizontal component $q_{2}$, arising as a result of the movement of oil products along the curved section of the pipeline. The dynamic smoothing method has been applied in order to assess the load rate $q_{2}[3,4]$.

\section{Research methods}

Let us consider the auxiliary problem. According to the geodetic data the pipeline axis position is known. On the basis of elastic rod equilibrium equations [13; 14] and the use of the d'Alembert principle we obtained homogeneous nonlinear equations of motion of the rod in a Cartesian coordinate system: 


$$
\begin{aligned}
& m \ddot{x}_{2}+\mu_{11} \dot{x}_{2}+\mu_{12} \dot{x}_{3}-\cos \varphi \frac{N^{\prime}+\varphi^{\prime} Q}{a}-\sin \varphi \frac{Q^{\prime}-\varphi^{\prime} N}{a}=0, \\
& m \ddot{x}_{3}+\mu_{12} \dot{x}_{2}+\mu_{22} \dot{x}_{3}-\sin \varphi \frac{N^{\prime}+\varphi^{\prime} Q}{a}+\cos \varphi \frac{Q^{\prime}-\varphi^{\prime} N}{a}=0 .
\end{aligned}
$$

In these equations the sign "' " denotes the derivative with respect to the Lagrangian coordinate of the point of the rod axis; the signs " " and " " stand for the first and the second time derivatives, $\mathrm{m}-$ mass per unit length of the pipeline with the product, $x_{2}$ and $x_{3}-$ Cartesian coordinates; $\varphi$ - the angle between the pipe axis and the $x_{3}$ axis; $N$ - the axial force, $N=D \varepsilon, \varepsilon=a-1-$ axial deformation of the pipe; $D=E \cdot A-$ stiffness in tension, $E-$ modulus of the pipe elasticity; $A$ - cross sectional area of the pipe; $Q=H \cdot \varphi$ " - shear force, $H=E \cdot J-$ pipe bending stiffness, $J-$ second area moment. Values $a$ and $\varphi$ are determined by known geodetic data - coordinates $x_{2}^{\prime}, x_{3}^{\prime}$ from the equations $x_{2}^{\prime}=a \cos \varphi, \quad x_{3}^{\prime}=a \sin \varphi$.

Coefficients of resistance $\mu_{i j}$ are:

$\mu_{11}=\mu_{\tau} \cos ^{2} \varphi+\mu_{n} \sin ^{2} \varphi$,
$\mu_{22}=\mu_{\tau} \sin ^{2} \varphi+\mu_{n} \cos ^{2} \varphi$,
$\mu_{12}=\left(\mu_{n}-\mu_{\tau}\right) \sin \varphi \cos \varphi$,

where $\mu_{\tau}$ and $\mu_{n}$ - known resistivity coefficients for movement along the tangent and the normal to the pipe axis respectively.

Let's introduce the boundary conditions: $x_{2}(0, t)=x_{0}, x_{3}(0, t)=y_{0}, x_{2}(l, t)=x_{l}$, $x_{3}(l, t)=y_{l}, \varphi(0, t)=\varphi_{0}, \varphi(l, t)=\varphi_{l}$.

The initial conditions: at time $\mathrm{t}=0 \quad x_{2}(0)=x_{0}(0), x_{3}(0)=y_{0}(0)$ - the initial form of a rod, $\dot{x}_{2}(0)=0, \dot{x}_{3}(0)=0$ - the initial velocities.

Transverse and longitudinal forces $q_{2}$ и $q_{3}$ are determined at any time by the formulas:

$q_{2}=\cos \varphi \frac{N^{\prime}+\varphi^{\prime} Q}{a}+\sin \varphi \frac{Q^{\prime}-\varphi^{\prime} N}{a}, \quad q_{3}=\sin \varphi \frac{N^{\prime}+\varphi^{\prime} Q}{a}-\cos \varphi \frac{Q^{\prime}-\varphi^{\prime} N}{a}$.

The system of equations (3) is solved by the finite difference method with the use of the "cross" explicit difference scheme [15]. The derivatives of $x_{3}$ coordinate are approximated by finite-difference formulas of the 2 nd order accuracy.

The system of equations (1) is solved with a modified finite element method.

Triangular finite elements have been taken as finite elements [8; 16]. The movements inside the triangle elements are described by linear dependencies through the nodal displacements $\boldsymbol{u}=\left(u_{1}, u_{2}\right)$ :

$u_{k}=\frac{1}{2 \Delta}\left[\left(p_{i}+d_{i} x_{1}+n_{i} x_{2}\right) u_{k}^{i}+\left(p_{j}+d_{j} x_{1}+n_{j} x_{2}\right) u_{k}^{j}+\left(p_{m}+d_{m} x_{1}+n_{m} x_{2}\right) u_{k}^{m}\right]$,

$p_{i}=x_{1}^{j} x_{2}^{m}-x_{1}^{m} x_{2}^{j}, \quad n_{i}=x_{1}^{m}-x_{2}^{j}, \quad d_{i}=x_{2}^{j}-x_{2}^{m}, \quad k=1,2$.

Nodal displacements $u_{k}^{i}, u_{k}^{j}, u_{k}^{m}$ are the desired values.

When taking the integral over the area of the triangular element there appear definite integrals $\int_{S} x_{1} d S$ and $\int_{S} x_{2} d S$. 


\section{TPACEE-2016}

Consider taking one of the integrals on the basis of the well-known theorem on the static moment of area according to which the static moment equals the area of a triangle $\Delta$ times $x_{c}$ distance to the center of gravity of the triangle, which coincides with the intersection of its medians:

$$
\int_{S} x_{1} d S=x_{1 c} \cdot \Delta, \quad x_{1 c}=\frac{x_{1}^{i}+x_{1}^{j}+x_{1}^{m}}{3}, \quad x_{2 c}=\frac{x_{2}^{i}+x_{2}^{j}+x_{2}^{m}}{3} \text {. }
$$

Here is a system of linear algebraic equations of the finite element method for finding the unknown nodal displacements $\{\delta\}=\left(u_{1}^{i}, u_{2}^{i}, u_{1}^{j}, u_{2}^{j}, u_{1}^{m}, u_{2}^{m}\right)$ [8]:

$\left(\left[k_{1}\right]+\left[k_{2}\right]\right)\{\delta\}=\{F\}$,

where:

$$
\begin{aligned}
& {\left[k_{1}\right]=\frac{1}{4 \Delta}\left(\begin{array}{ccc}
d_{i} & 0 & n_{i} \\
0 & n_{i} & d_{i} \\
d_{j} & 0 & n_{j} \\
0 & n_{j} & d_{j} \\
d_{m} & 0 & n_{m} \\
0 & n_{i} & d_{m}
\end{array}\right) \cdot\left(\begin{array}{ccc}
\lambda \frac{1-v}{v}+b_{1} & 2 G \frac{v}{1-2 v} & 0 \\
2 G \frac{v}{1-2 v} & \lambda \frac{1-v}{v}+b_{2} & 0 \\
0 & 0 & G
\end{array}\right) \cdot\left(\begin{array}{cccccc}
d_{i} & 0 & d_{j} & 0 & d_{m} & 0 \\
0 & n_{i} & 0 & n_{j} & 0 & n_{m} \\
n_{i} & d_{i} & n_{j} & d_{j} & n_{m} & d_{m}
\end{array}\right)} \\
& {\left[k_{2}\right]=\frac{1}{4 \Delta}\left(\begin{array}{ccc}
f_{i} & 0 & 0 \\
0 & f_{i} & 0 \\
f_{j} & 0 & 0 \\
0 & f_{j} & 0 \\
f_{m} & 0 & 0 \\
0 & f_{m} & 0
\end{array}\right) \cdot\left(\begin{array}{ccc}
c_{1} & 0 & 0 \\
0 & c_{2} & 0 \\
0 & 0 & 0
\end{array}\right) \cdot\left(\begin{array}{cccccc}
d_{i} & 0 & d_{j} & 0 & d_{m} & 0 \\
0 & n_{i} & 0 & n_{j} & 0 & n_{m} \\
n_{i} & d_{i} & n_{j} & d_{j} & n_{m} & d_{m}
\end{array}\right) \text {, }} \\
& f_{i}=p_{i}+d_{i} x_{1 c}+n_{i} x_{2 c}, f_{j}=p_{j}+d_{j} d x_{1 c}+n_{j} x_{2 c}, f_{m}=p_{m}+d_{m} x_{1 c}+n_{m} x_{2 c} \text {, } \\
& \int_{S} d S=\Delta=\frac{1}{2}\left|\begin{array}{lll}
1 & x_{1}^{i} & x_{2}^{i} \\
1 & x_{1}^{j} & x_{2}^{j} \\
1 & x_{1}^{m} & x_{2}^{m}
\end{array}\right| \text {. }
\end{aligned}
$$

\section{Numerical results}

The system of equations (1) with the boundary conditions (2) was solved by a modified method of finite elements. The triangular finite elements were chosen as finite element.

The value of the intensity of the horizontal force $q_{2}=0.005 \mathrm{MN} / \mathrm{m}$

Acting at a distance of $10 \mathrm{~m}$ along $x_{3}$ axis ; own weight of the pipe $q_{1}=0.0015 \mathrm{MN} / \mathrm{m}$ (Fig. 1) 


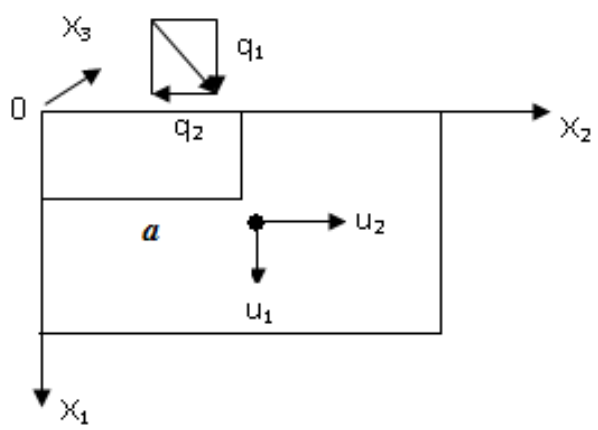

Fig. 1. Forces acting on the water-saturated base.

The mechanical characteristics of the soil

$$
\begin{aligned}
& E_{s}=5.2 \mathrm{MPa}, E_{l}=3.27 \mathrm{MPa}, \\
& v=0.3, \aleph=0.52,
\end{aligned}
$$

the radius of the pipeline $-0.3 \mathrm{~m}$.

The Fig. 2 shows the horizontal displacement of soil skeleton particles at $x_{1}=0 \mathrm{M}, x_{2}=1 \mathrm{~m}$.

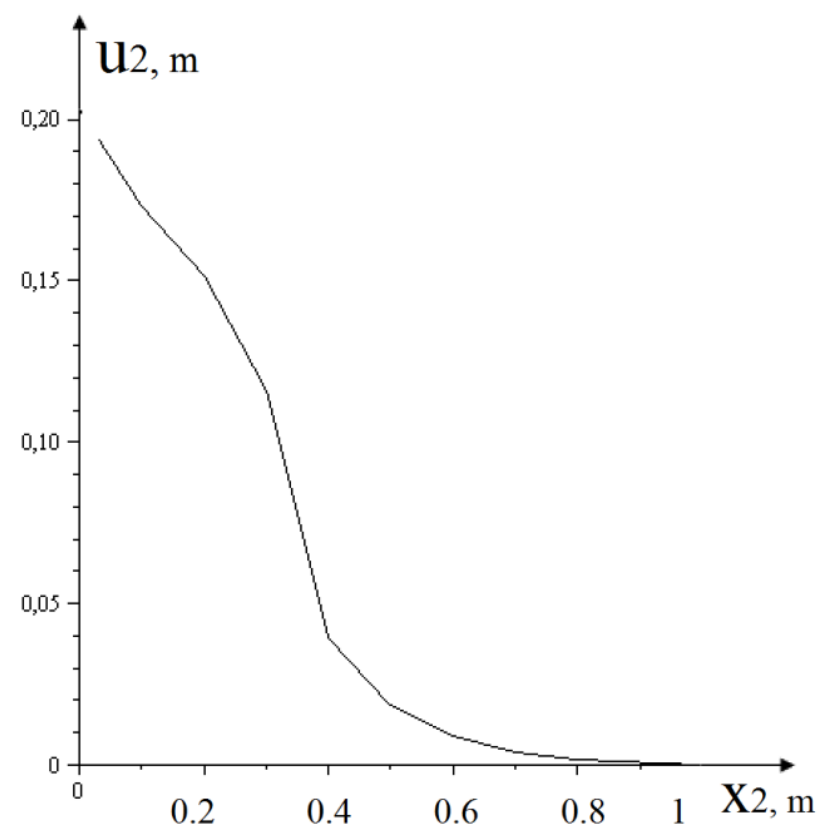

Fig.2. Horizontal displacement of soil skeleton particles 


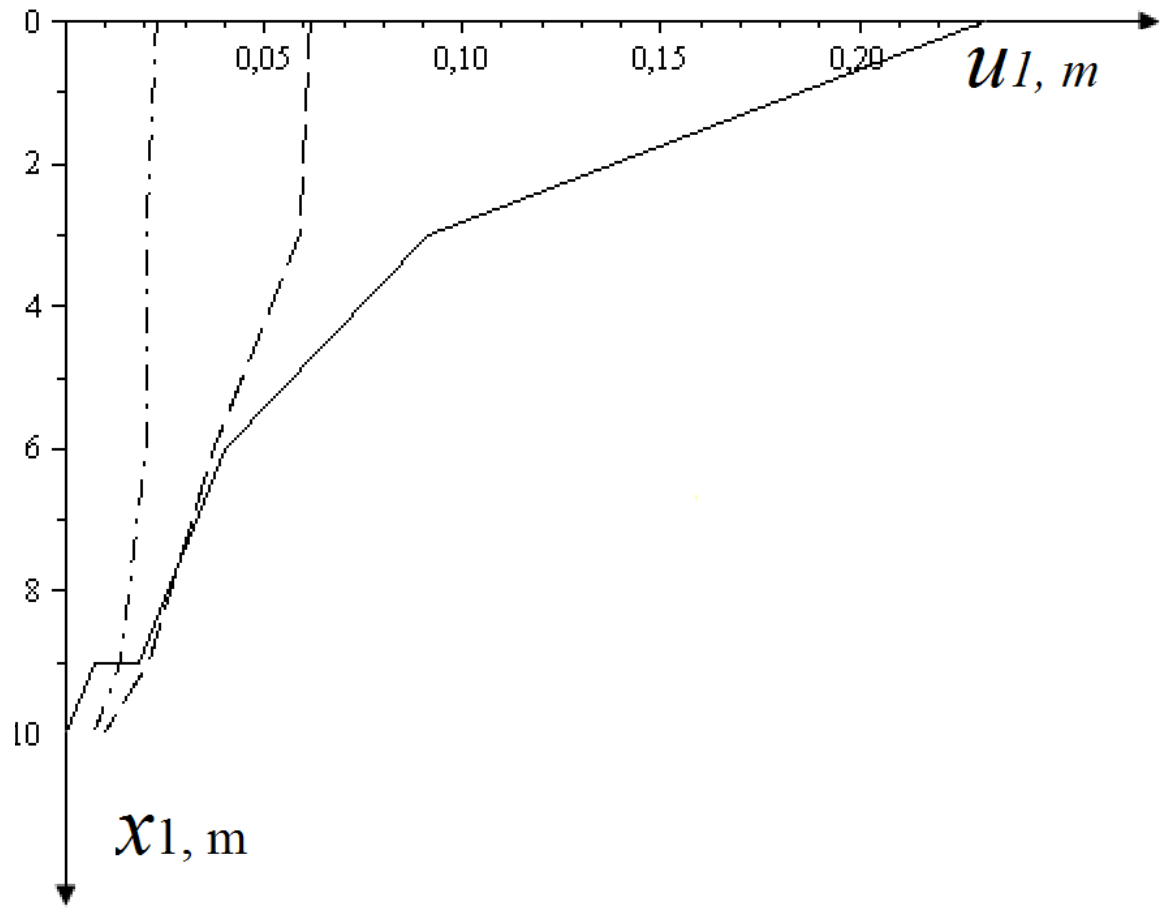

Fig. 3. Vertical displacement for different cross-sections.

Fig. 3 shows the graphs for vertical movement for $x_{2}=0$, at the distance for $x_{2}=0$, at the distance $x_{2}=0.3 \mathrm{~m}, x_{2}=0.9 \mathrm{~m}$ from $x_{1}$ axis

The maximum horizontal displacements were $0.2 \mathrm{~m}$, the minimum ones $-0.03 \mathrm{~m}$.

The solution method solves the problem of determining the longitudinal and transverse forces of the interaction of the pipeline with the ground for sufficiently long pipeline sections. Transverse bends lead to the loss of stability of plane shape of the bending. The proposed model and solution algorithm allow us to take account of the possibility of the effect of thermal stresses in the pipeline on the strength of the interaction with the ground, but the article does not deal with this question. When solving the problem the maximum load of the pipeline on the ground was determined and it accounted for $q=0.0166 \mathrm{MN} / \mathrm{m}$

\section{Conclusion}

The obtained results can be used in the design and the calculation of sediment of engineering pipeline transportation facilities being built on weak grounds in determining the residual life of operated pipelines or in the processing of seismic results. The modified finite element method allows us to determine the stress and strain state of the base of the water-saturated soil under the action of external forces caused by the influence of the pipeline.

The solution to the problem of stress-strain state of a weak base will allow us to predict the maximum permissible values of the base strain and to consider and adopt measures to improve the safety of its construction and operation at the design stage. 


\section{References}

1. T. Maltseva, S. Dorofeev, T. Selivanov, Izvestija visshikh uchebnikh zavedenij. Neft i gaz, 1, 59-63 (2012)

2. T. Maltseva, A. Nabokov, A. Chernykh, Procedia Engineering, 117, 239-245 (2015)

3. A. Kraev, Vestnik Tomskogo arkhitekturno-stroitelnogo universiteta, 4, 146-151 (2008)

4. V. Baj, T. Maltseva, A. Kraev, Nauchno-tehnicheskij vestnik Povolzh'ja, 5, 108-111 (2014)

5. V. Baj, A. Kraev, Vestnik grazhdanskih inzhenerov, 3, 107-110 (2014)

6. L. Maltsev, T. Maltseva, A. Minaeva, A. Nabokov, Nauchno-tehnicheskij vestnik Povolzh'ja, 2, 234-238 (2012)

7. T. Maltseva, Matematicheskaja teorija vodonasyshhennogo grunta (Vektor Buk, Tjumen', 2012)

8. L. Maltsev, T. Maltseva, T. Saltanova, Problemi prochnosti i plastichnosti, 68, 181 (2006)

9. V. Baj, L. Maltsev, T. Maltseva, A. Nabokov, A. Demin, R.F. Patent 2213952. (2002)

10. L. Maltsev, T. Maltseva, A. Demin, Izvestija vysshih uchebnyh zavedenij. Neft' i gaz, 2, 96-102 (2001)

11. S. Dorofeev, N. Nesterovich, Sbornik nauchnikh trudov TumGU, 11, 65-70 (2009)

12. S. Dorofeev, N. Nesterovich, Sbornik nauchnikh trudov TumGU, 11, $71-75$ (2009)

13. S. Toropov, S. Dorofeev, E. Sapozhnikov, Izvestiya vysshikh uchebnykh zavedenij. Neft' i gaz, 3, 49-53 (2003)

14. E. Grigolyuk, V. Shalashilin, Problemy nelinejnogo deformirovaniya, (Nauka, Moscow, 1988)

15. N. Kalitkin, Chislennie metodi (Nauka, Moscow, 1978)

16. T. Maltseva, T. Saltanova, Vestnik TumGU, 49-56 (2007) 